\title{
Death and dying in the US: the barriers to the benefits of palliative and hospice care
}

\section{Albert J Finestone Gail Inderwies}

School of Medicine, Temple University, Philadephia, PA, USA
Correspondence: Albert J Finestone Director, Institute on Aging, Geriatric Education Center, Temple University, Philadephia PA, USA

Tel + I 2 I5 707474 I

Fax +I 2157073675
In August 2006, after a trip to the New Jersey Shore, Peggy was having great difficulty catching her breath. In consultation with her children, Peggy decided that she was ready for hospice care. But, she did not want to relinquish her independence just because shortness of breath and a weakening heart overtook her daily stride. However, a single episode at home had thrown Peggy into crisis. Since Peggy lived alone, hospice care at home presented a host of challenges including safety and how to manage her unstable cardiopulmonary condition. Peggy was an ideal candidate for the hospice's TeleCare (see box) monitoring program which provided a passive monitoring system, a medication dispenser, and vital signs monitoring for blood pressure, weight, and blood oxygen levels. In addition, the hospice authorized routine draws of BNP (beta naturetic peptide) and BMP (basic metabolic profile) with GFR (glomerular filtration rate) to manage her symptoms aggressively. Medications were adjusted accordingly to maximize quality of life and minimize symptoms. Though some would consider this treatment aggressive, it was the aggressive treatment of Peggy's symptoms that allowed for an extended quality of life. There was sufficient evidence to support this action based on the concept of risk and reward, especially as there was a minimum of invasive therapies required. In Peggy's case she went from being homebound and short of breath to living her life up to her final days.

TeleCare monitoring enabled a hospice patient like Peggy to not only live independently, but also to leverage the hospice staff's ability to care for her. The nurse case manager could identify Peggy's changing medical status for immediate intervention before symptoms escalated into a crisis. Making more informed and timely adjustments to Peggy's treatment protocol allowed for intensive treatment of her symptoms and improved her overall quality of life. In Peggy's case TeleCare monitoring played an important part in her living longer, more comfortably, and with peace of mind. Peggy had witnessed her father suffocate with emphysema and she feared that would also be her fate. But with her hospice care augmented with Telecare, Peggy's children agreed that their mother never struggled to breathe. Peggy lived at home for another 2 months, and it was there she was able to celebrate her last birthday, close to her children and family.

\section{What is hospice?}

The hospice concept was pioneered in 1967 by English physician, nurse and social worker Dr. Dame Cicely Saunders. In the US, the hospice movement emerged in the mid 1970s. In 1982, Congress initiated the hospice benefit under TEFRA (Tax Equity and Fiscal Responsibility Act), a landmark public policy decision to include hospice care in the Medicare program. Hospice core services include professional nursing care, personal assistance with activities of daily living, various forms of rehabilitation therapy, dietary counseling, psychosocial and spiritual counseling for both patient and family, volunteer 
services, respite care, provision of medical drugs and devices necessary for palliative care, and family bereavement services after the patient's death. Hospice care is provided by an interdisciplinary care team comprising nurses, social workers, pastoral counselors, nursing assistants, and other health professionals under the management of the patient's own primary care physician or one affiliated directly with hospice program.

Care for the dying is a complex enterprise that must involve multiple professionals and nonprofessionals. The physical, emotional, and social needs of the dying person are addressed by acknowledging the fear, anxiety, loneliness, and isolation that is experienced during an end-stage illness.

Most elderly patients are eligible for Medicare Hospice benefits (MHB). For an individual to be elected for hospice care, a physician must certify that the patient is likely to die within 6 months if the terminal disease follows its anticipated course. The patient or the patient's representative in turn agrees to waive all other Medicare coverage related to their terminal illness under part " $A$ " which is Medicare Hospital Benefits. A hospice patient's primary physician can bill under Medicare part "B". Hospice patients may be hospitalized for a brief period of time. Medicare payment for hospice requires that a patient be reassessed periodically, initially after each of the first two 90-day periods, and then 60 days after that to document continued decline in condition and determine whether hospice care continues to be appropriate.

\section{The state of end-of-life care in the US}

Despite the powerful and valuable Medicare Hospice Benefit, there is a persistent culture of ICU (Intensive Care Unit) hospitalization for end-of-life care for these patients (Seferian and Afessa 2006), an expensive and often futile strategy. In my experience (AJF) nursing homes are reluctant to have patients die while under their care. Hence, when a patient with a terminal illness in a nursing home becomes close to death, emergency services are called, and the patient is transferred to the nearest hospital and admitted to the ICU where expensive and futile services are provided. Hospice choice will avoid this unnecessary detour. It has been estimated that Medicare payments made to beneficiaries in the last year of life are almost 7 times greater than those made for all Medicare beneficiaries (Lubitz and Riley 1993). In addition to the recognition of overuse of technology in terminally ill patients, there is also a growing perception of a significant lack of symptom control and psychological support for patients who die in conventional hospital settings (Solomon et al 1993; SUPPORT Principal Investigators 1995; Lynn et al 1997; Reynolds et al 2002; Teno et al 2004).

\section{Benefits of hospice care}

There is ample evidence to support a higher quality of life in hospice patients compared with terminally ill patients in the hospital setting. Numerous studies evaluating quality of end of life in settings other than the hospital show that family members are consistently more likely to report a favorable dying experience of the decedent when hospice or palliative care is chosen, compared with hospitalization (Dawson 1991; Hanson et al 1997; Nolen-Hoeksema et al 2000; Teno et al 2004). Here is growing evidence that hospice provides high quality care with high consumer satisfaction (Casarett and Quill 2007). Research has suggested that for certain diagnosis such as CHF, compared with patients who do not choose hospice care, hospice patients live longer for an average of 29 days (Connor et al 2007), and that hospice care may be associated with a modest cost-saving (Pyenson et al 2004).

\section{Underutilization of hospice in the US}

Despite the clear advantages in quality of life for terminally ill patients, and the cost benefits associated with palliative and hospice care, the decision to utilize hospice is made by only an estimated fraction of the patients who stand to benefit. Only approximately $20 \%-25 \%$ of people who die in the US utilize hospice services (Foley and Gelbard 2001; Hanson 2004). The median utilization of hospice is only 22 days, and over one-third of hospice patients receive fewer than 8 days of services (Russell and LeGrand 2006). Ten percent of hospice patients are enrolled in the last 24 hours of their life (NHPCO 2006; National Trend Study 2004). Over one-third of patients receiving hospice care in 2002 were over the age of 85 years, and the overwhelming majority (82\%) were white (Connor et al 2004). There is therefore clear evidence that hospice is poorly utilized in the US, and that this underutilization is at least partially dependent upon demographic factors including race or ethnicity, misconceptions of financial and eligibility requirements, and difficulty in discussing or accepting hospice as a treatment option.

\section{The demographic divide in the US}

Many studies report the observation that minority groups are less likely than white Americans to benefit from hospice or palliative care. African Americans and Latinos are more likely to die at home than European Americans, but are significantly less likely to receive hospice care (Enguidanos et al 2005). During the period 1995 to 2001, the use of hospice services by African Americans and Latinos was significantly less than by European Americans, and, though European American use 
of hospice increased during this period, African American use actually decreased (Colon and Lyke 2003). The difference appears to be when end-of-life decision making is initiated. When ethnic groups who choose to use hospice were compared in one study, there were no differences between European Americans and Latino patients in average duration of hospice use, and African Americans utilized hospice, on average, longer than either. Furthermore, there was no greater likelihood that services would be terminated prematurely among ethnic minorities when compared with European Americans (Colon and Lyke 2003; Johnson et al 2005). An important recent report by Kapo and co-investigators suggests that the return rate to hospice may be lower in African Americans compared with all other users (Kapo et al 2005). Elderly minorities in this group were more likely to die in an inpatient setting than their European counterparts (Jonson et al 2005).

A large number of factors have been identified for the underutilization of hospice by ethnic minorities and greater utilization of inpatient settings by elderly minorities. Some of the differences in the making of end-of-life decisions may be related to associated or indirect factors, such as differences in the availability of a full-time caregiver, in marital status, in general economic or educational status, or language use (Colon and Lyke 2003). However, a large number of cultural and social factors, that are race or ethno-specific, have also been identified as possible determinants of hospice underutilization. These include a lack of knowledge of hospice, cultural, or religious beliefs about end of life and death, the desire for autonomy, and, importantly, perceptions and mistrust of healthcare and healthcare professionals (especially among African Americans) (Burrs 1995; Gordon 1996; Reese et al 1999; Born et al 2004; Torke et al 2005; Winzelberg et al 2005; Duffy et al 2006; Rhodes et al 2006). These ethnic, social, and cultural complexities in end-of-life perceptions place a burden on health-care professionals to remain sensitive to diverse factors during clinical decision-making. However, it is poorly understood how physicians, nurses, and other healthcare professionals working specifically within racially diverse, low-income communities see their role in this process.

\section{Barriers to hospice use}

Because life expectancy for patients with most end-stage diseases cannot be predicted with specificity, there has been recent focus on how the Medicare mandated assignment of a 6-month time frame as discussed previously has itself become a barrier to care (Casarett and Quill 2004). Because the culture of medicine is that physicians and other health professional are trained to prolong life, referral to hospice maybe viewed as a medical failure or depriving patients of hope. There are consumer barriers to access to hospice, with various attitudes, and misinformation, including that they must forgo all treatment. The National Hospice Foundation reveals $75 \%$ of Americans do not know that hospice care can be provided in the home and 90\% do not realize that hospice care is fully covered through Medicare.

\section{$100 \%$ mortality in this world}

The association of hospice with death is a major impediment to hospice enrollments as fear of death is a pervasive human emotion. Palliative care and hospice patients are often not capable of engaging in the types of interactions required to make end-of-life choices independently, and the influence of others is crucial both physically and psychologically. The role of family in the choice of, and evaluation of, hospice care has long been recognized (Connor et al 2005). However, next to the influence of friends and relatives, healthcare professionals are logically the most influential group during end-of-life decisions. It has been suggested that quality of end-of-life care results when, among other things, health-care professionals promote shared decisionmaking (Teno et al 2001). However, a great deal of evidence exists to suggest that the influence of healthcare professionals on decision-making in ethnic minorities may be significantly different than their role among white patients and their families, resulting from a substantial cultural mistrust (Cort 2004; Welch et al 2005). It has been recognized that there is a greater need for healthcare professionals to be cognizant of diverse cultural and social issues that relate to end of life decision-making, such as distrust of the medical system, methods for communicating news about life-threatening illness, autonomy, and attitudes toward advanced directives (a number of guidelines are available, including [Searight and Gafford 2005]). There is a strong precedent for using patient- and family-based surveys to inform healthcare providers on strategies and possible improvements (Lanford et al 2001; Jenkinson et al 2002). What is needed are similar strategies that directly measure the perception and roles of various healthcare professionals in the clinical decision-making process as it pertains to end of life and palliative care.

\section{Conclusion}

Providers and patients must recognize that death is inevitable. Hospice should not be viewed as care of last resort but rather as an alternative option that comes after aggressive treatment of the terminal illness has failed.

Unfortunately, many Americans have their access to hospice and other forms of palliative care blocked by lack of information, misunderstanding, financial limitation, and other less tangible factors including fear. This summary has 
addressed some of the issues preventing more wide spread use of this valuable palliative and hospice care option.

\section{Telecare}

In a recent pilot program sponsored by Keystone Hospice in Wyndmoor, PA, USA, the Telecare Program reduced the risks of providing services to vulnerable elderly individuals with the use of simple monitoring and medication compliance technology. The program combines technology to monitor activity and ambient temperature in the home, track and dispense medication doses, and monitor vital signs with home care support.

The approach is three-tiered:

- Passive activity monitoring: Wireless motion detectors strategically placed in the home track functional activities of daily living and ambient temperature. Getting out of bed, eating, using the bathroom, taking medication, tasks necessary for independent living, and ambient temperature. A baseline analysis of the individual's safe independent status is recorded. When activity deviates from the known pattern, alerts are sent to caregivers. Data aremonitored 24 hours a day by a call center for emergency situations such as suspected bathroom fall, lack of wake up, or extremes in ambient temperature.

- Medication management: Using the MD2, the device that reminds, dispenses, monitors, and safeguards daily doses of medications. The MD2 holds up to sixty doses of medication and is a reliable and effective device for increasing medication adherence and reducing medication error. Caregivers are able to monitor compliance and are alerted to missed doses of medication.

- Vital signs monitoring: Low cost tools (glucose meter, blood pressure, scale) are adapted to send measurements over phone lines to physician and care manager. These measurements create a longitudinal record for accuracy of disease assessment. When monitored with more frequency, trending is evident. Intervention by the physician or care manager can occur before crisis.

Benefits identifed during the course of the project were:

- Move care from facilities into the home, decrease use of expensive services, emergency room visits, inpatient stays.

- Decreased costs long-term care, avoid precipitous nursing home placement, increased safety, quality of life, and independence.

A case in point - Peggy.

\section{References}

Born W, Greiner K, et al. 2004. Knowledge, attitudes, and beliefs about endof-life care among inner city African Americans and Latinos. J Palliat Med, 7:247-56.

Burrs F. 1995. The African American experience: breaking the barriers to hospices. Hosp J, 10:15-18.

Casarett D, Quill T. 2007. "I'm not ready for hospice”: strategies for timely and effective hospice discussions. Ann Intern Med, 146:443-9.

Colon M, Lyke J. 2003. Comparison of hospice use and demographics among European Americans, African Americans, and Latinos. Am J Hosp Palliat Care, 20:182-90.

Connor S, Tecca M, et al. 2004. Measuring hospice care: The National Hospice and Palliative Care Organization National Hospice Data Set. J Pain Symptom Manage, 28:316-28.

Connor S, Pyenson B, et al. 2007. Comparing hospice and non-hospice patient survival among patients who die within a three-year window. J Pain Symptom Manage, 33:238-46.

Connor S, Teno J, et al. 2005. Family evaluation of hospice care: results from voluntary submission of data via website. J Pain Symptom Manage, 30:9-17.

Cort M. 2004. Cultural mistrust and use of hospice care: challenges and remedies. J Palliat Med, 7:63-71.

Dawson N. 1991. Need satisfaction in terminal care settings. Soc Sci Med, 32:83-7.

Duffy S, Jackson F, et al. 2006. Racial/ethnic preferences, sex preferences, and perceived discrimination related to end of life care. J Am Geriatr Soc, 54:150-7.

Enguidanos S, Yip J, et al. 2005. Ethnic variation in site of death of older adults dually eligible for Medicaid and Medicare. $J$ Am Geriatr Soc, $53: 1411-16$

Foley K, Gelbard HE. 2001. Institute of Medicine Report: Improving palliative care for cancer. Washington, DC: National Academy Press.

Gordon A. 1996. Hospice and minorities: a national study of organizational access and practice. Hosp $J$, 11:49-70.

Hanson L. 2004. Palliative care: Innovation in care at the end of life. North Carolina Medical Journal, 65:202-8.

Hanson L, Danis M, et al. 1997. What is wrong with end of life care? Opinions of bereaved family members. J Am Geriatr Soc, 45:1339-44.

Jenkinson C, Coulter J, et al. 2002. Patients' experiences and satisfaction with health care: results of a questionnaire study of specific aspects of care. Qual Saf Health Care, 11:335-9.

Johnson K, Kuchibhatala M, et al. 2005. Ethnic differences in the place of death of elderly hospice enrollees. J Am Geriatr Soc, 53:2209-15.

Kapo J, Macmoran H, et al. 2005. Lost to follow-up: Ethnic disparities in continuity of hospice care at the end of life. J Palliat Med, 8:603-8.

Koppen J. 2007. Thoughts on the afterlife among US adults 50+. AARP Magazine.

Lanford A, Clausen R, et al. 2001. Measuring and improving patients' and families' perceptions of care in a system of pediatric hospitals. Jt Comm J Qual Improv, 27:415-29.

Lubitz J, Riley G. 1993. Trends in Medicare payments in the last year of life. $N$ Engl J Med, 328:1092-6.

Lynn J, Teno J, et al. 1997. Perceptions by family members of the dying experience of older and seriously ill patients. Ann Intern Med, 126:97-106.

NHPCO Facts and Figures: Hospice Care in America. 2006. National Hospice and Palliative Care Organization.

National Hospice and Palliative Care Organization National Data Set: National Trend Study. 2004.

Nolen-Hoeksema S, Larson J, et al. 2000. Predictors of family members' satisfaction with hospice. Hosp J, 15:29-48.

Pyenson B, Connor S, et al. 2004. Medicare cost in matched hospice and non-hospice cohorts. J Pain Symptom Manage, 28:200-10.

Reese D, Ahern R, et al. 1999. Hospice access and use by African Americans: Addressing cultural and institutional barriers through participatory action research. Soc Work, 44:549-59. 
Reynolds K, Henderson M, et al. 2002. Needs of the dying in nursing homes. $J$ Palliat Med, 5:895-901.

Rhodes R, Teno J, et al. 2006. Access to hospice for African Americans: Are they informed about the option of hospice? J Palliat Med, 9:268-72.

Russell K, LeGrand S. 2006. I'm not that sick! Overcoming the barriers to hospice discussions. Cleveland Clinic J Med, 73:517-24.

Searight H, Gafford J. 2005. Cultural diversity at the end of life: issues and guidelines for family physicians. Am Fam Physician, 71:515-22.

Seferian E, Afessa B. 2006. Adult intensive care use at the end of life: a population based study. Mayo Clinic Proc, 81:896-901.

Solomon M, O'Donnell L, et al. 1993. Decisions near end of life: professional views on life-sustaining treatments. Am J Public Health, 83:14-23.

SUPPORT Principal Investigators. 1995. A controlled trial to improve care for seriously ill hospitalized patients. The study to understand prognoses and preferences for outcomes and risks of treatments (SUPPORT). JAMA, 274:15918 (Erratum in: JAMA, 1996. 275:1232).
Teno J, Casey V, et al. 2001. Patient-focused, family-centered end-of-life medical care: views of the guidelines and bereaved family members. J Pain Symptom Manage, 22:738-51.

Teno J, Clarridge B, et al. 2004. Family perspectives on end-of-life care at the last place of care. JAMA, 291:88-93.

Torke A, Garas, et al. 2005. Medical care at the end of life: views of African American patients in an urban hospital. J Palliat Med, 8:593-602.

Welch L, Teno J, et al. 2005. End-of-life care in black and white: race matters for medical care of dying patients and their families. $J \mathrm{Am}$ Geriatr Soc, 53:145-53.

Winzelberg G, Hanson L, et al. 2005. Beyond autonomy: diversifying end-of-life decision-making approaches to serve patients and families. $J$ Am Geriatr Soc, 53:1046-50. 